\title{
ZUR GLIEDERUNG DES JUNGPLEISTOZÄNS IM GRENZBEREICH VON LINTH- UND RHEINSYSTEM
}

René HantKe

\section{DIE «MITTELTERRASSENSCHOTTER»IM GLATTAL UND IM ZÜUICHSEETAL}

Bei der Überprüfung der bisher in der Umgebung von Zürich als «Mittelterrassenschotter» zusammengefaßten Bildungen und ihrer Parallelisation mit analogen Bildungen im Aare/Rhone-System zeigte sich (R. HANTKE 1959a), daß diese Schotter recht komplexer Natur sind. Bei Gossau (Zürich) und am Buechberg am Zürcher Obersee treten über einwandfreier Grundmoräne (1) 20-40 m mächtige eisrandnahe Deltaschotter (2) zutage. Über einer Übergußschicht (3) schalten sich bei Gossau limnische Ablagerungen, ein Gyttjahorizont mit Pollen wärmeliebender Laubhölzer (4), ein. Dann folgt ein 1,5-2 m mächtiger Schieferkohlenhorizont (5), der bei Gossau von einer bis $4 \mathrm{~m}$ mächtigen glazifluviatilen Deckschicht (6) überlagert wird. Darüber liegt mit scharfer Erosionsdiskordanz Grundmoräne (9).

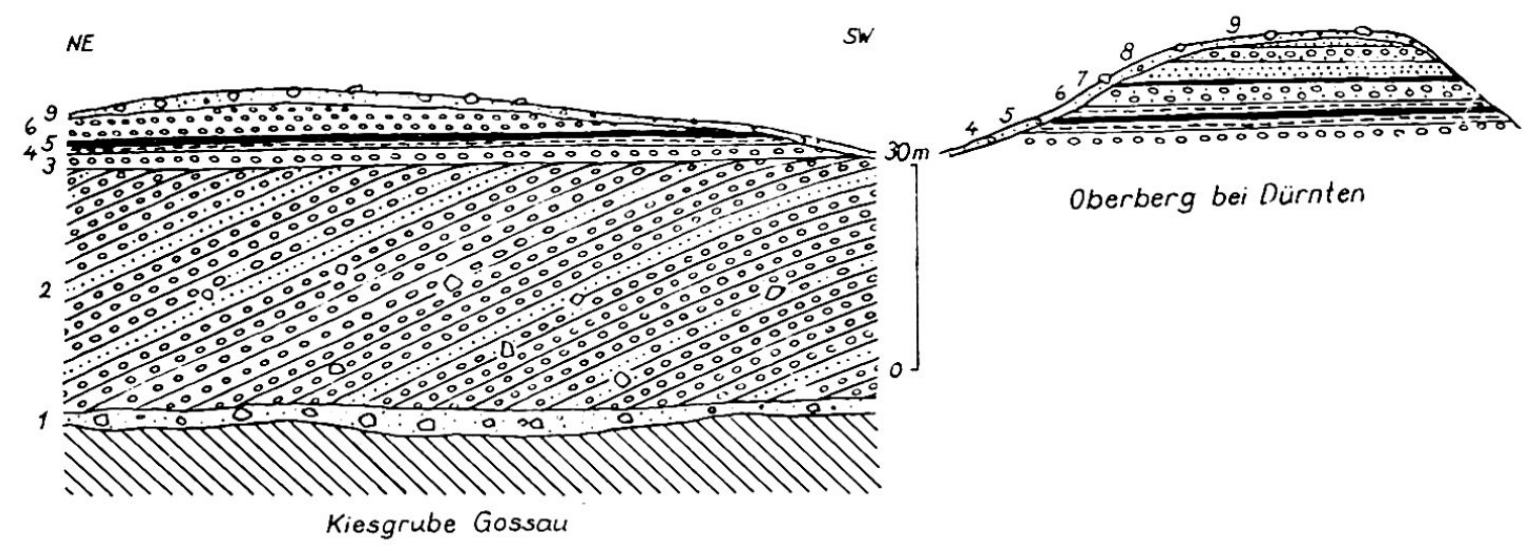

Bei Dürnten, etwas mehr gegen den Beckenrand, glaubte E. BAumberger (in E. Baumberger et alii 1923: Taf. 21) innerhalb dieses dort etwa $30 \mathrm{~m}$ mächtigen Schotterkomplexes mit Sicherheit 4 verschiedene Kohlenhorizonte auseinanderhalten zu können. Ob bei Dürnten effektiv 4 verschiedenaltrige Flöze vorliegen, scheint allerdings fraglich, konnte doch BAUMBERGER im selben Schürfprofil maximal zwei, durch Schotter getrennte Kohlenniveaus feststellen. Auf Grund der Schichtfolgen ist es jedoch wahrscheinlich, daß die oberste, am Oberberg NW Dürnten in $535 \mathrm{~m}$ auftretende Kohlenschicht über den sandigen Schottern mit Elephas (Palaeoloxodon) antiquus FALc. und Rhinoceros mercki JAEG. eine jüngere Ablagerung darstellt als der Schieferkohlenhorizont von Gossau, der wohl dem 1,5 m mächtigen Kohlenlager von Dürnten-Oberberg (Flöz II nach der Bezeichnung von E. BAUMBERGER) glcichgesetzt werden darf. Diese Parallelisation sollte jedoch unbedingt mit C ${ }^{14}$-Analysen überprüft werden.

Die beiden Profile von Gossau und von Dürnten lassen sich wie folgt deuten: (1) $=$ rißeiszeitliche Grundmoräne; $(2)=$ spätrißeiszeitliche Rückzugsschotter, die in einen vom Gletscher ausgekolkten, eisrandnahen See geschüttet wurden; (3) = das Seebecken ist praktisch mit Schottern gefüllt; (4) $=$ Wärmeoptimum des Interglazials; dürfte dem Seekreidehorizont von Gublen bei Uznach entsprechen, der Blattreste wärmeliebender Laubhölzer geliefert hat (cf. R. HANTKE 1959a: 10); (5) = 
limnische Kohlenbildung, die auf Grund des Fossilinhaltes bereits auf ein etwas kühleres und feuchteres Klima schließen läßt; $(6)=$ frühwürmeiszeitlicher Vorstoßschotter; $(7)=$ frühwürmeiszeitliche Schwankung (Göttweiger Interstadial ??), ( 8$)=$ erneute Kälterückschläge mit Gletschervorstößen, (9) = nach vorgängiger glazialer Erosion darüber abgelagerte hochwürmeiszeitliche Grundmoräne.

Frühwürmeiszeitliche Vorstoßschotter konnten im Glattal erst zur Ablagerung gelangen, als der anrückende Linthgletscher die Schwelle von Hombrechtikon erreicht hatte. Die höhere Schieferkohle dokumentiert ohne jeden Zweifel wieder eine etwas wärmere Periode, eine Schwankung innerhalb des frühwürmeiszeitlichen Gletschervorstoßes. $\mathrm{Zu}$ dieser Zeit wich offenbar der Gletscher nochmals hinter die Glattalschwelle zurück, so daß es im Glattal zu erneuter Kohlenbildung kommen konnte, die beim nächsten Vorstoß über die Molassebarre von Hombrechtikon wieder von lehmigen Sanden und sandigen Schottern eingedeckt wurde.

Dies wirft auch neues Licht auf die Frage nach der Entstehung der Alpenrandseen (cf. R. Stau b 1939). Mit dem Vorstoß über die Glattalschwelle wurde die seit der ausgehenden Riß-Eiszeit existierende Wanne des Zürichsees wieder von Gletschereis erfüllt und, da die Hauptschüttung offensichtlich durch das Glattal erfolgte, vor der Zuschüttung bewahrt.

Den Schottern des Glattales analoge Bildungen konnten neulich auch im Zürichseetal erkannt werden (R. HANTKE 1959b). Besonders instruktiv ist in dieser Hinsicht der von Grundmoräne bedeckte, stark verkittete Schotter von $W u l p$ im Küsnachter Tobel. Der schon von Alexander Wettstein (1885: 23) erwähnte Schotter liegt nach J. Hug (in J. Hug und A. Beilick 1934: 111) in einer etwa $400 \mathrm{~m}$ breiten, in die Molasse eingetieften Rinne. Da er ziemlich genau in der Höhenlage der Seitenmoränen des Zürich-Stadiums liegt, jedoch eindeutig von Grundmoräne überdeckt ist, scheint es naheliegend, diesen Schotter als glazifluviatile Ablagerung eines vorstoßenden frühwürmeiszeitlichen Gletschers zu betrachten, dessen Eisstand etwa demjenigen des Zürich-Stadiums entsprach (cf. R. Hantke 1959b). Nach Zürcher Terminologie müßte dieser Schotter als oberster und damit als jüngster «Mittelterrassenschotter» bezeichnet werden (cf. R. HANTKE 1959a).

\section{FRÜHWÜRMEISZEITLICHE SCHOTTER ZWISCHEN PFÄFFIKERSEE UND TÖSSTAL}

Eine durchaus analoge Schotterflur stellte ArMIN WEBER (1928) im Tößtal und in den südlichen Seitentälern, etwa $70 \mathrm{~m}$ über dem Talgrund der Töß, fest. Zufolge ihrer «hohen Lage» ordnete er sie der «hohen Terrasse»zu. Dem Gliederungsschema PEncks und BrüCKNers folgend, stellt er sie in die Riß-Eiszeit.

Bereits A. Weber beobachtete, daß diese Schotter im oberen Tößtal fast durchweg von mächtiger Grundmoräne unterteuft werden. Merkwürdigerweise nehmen die Schotterfluren ihren Anfang auf der Glattalseite in einer Höhenlage, die èbenfalls etwa einem Gletscherstand des Zürich-Stadiums entspricht. Auch sie wurden, wie der Schotter von Wulp, von gering mächtiger Grundmoräne bedeckt ${ }^{1}$ und in den zur Töß entwässernden Quertälern noch von deutlichen Wallmoränen gekrönt. Besonders eindrucksvoll ist die Stirnmoräne bei Bliggenswil NW Bauma. Dieser Wall wird von Max Stein (1948) dem «Alte Burg» (= Killwangen)-Stadium der Würm-Eiszeit zugeordnet. Die Grundmoräne unter den Schottern kann daher, zufolge ihrer Lage außerhalb des Maximalstadiums der Würm-Eiszeit, nur rißeiszeitlich sein.

Analoge Lagerungsverhältnisse treffen wir in den andern zur Töß entwässernden Talungen, so im Tal Hittnau-Saland (Killwangen-Stadium unterhalb Fischbach) und

1 Auf der Karte von A. Weber (1928) wird diese Grundmoräne allerdings zum Teil als *alluviale Anschwemmung " ausgeschieden. 
im Tal Russikon-Madetswil-Rikon (Killwangen-Stadium unterhalb Bläsimüli). A. Weber gibt auch im Tal von Gündisau und in den Tälern Rumlikon-TheilingenWeißlingen und Agasul-Weißlingen Schotter der «hohen Terrasse» an; doch liegen diese Schotter nicht auf Grundmoräne, sondern direkt auf Molasse. Ihre Gleichaltrigkeit mit den Schottern der übrigen Quertäler erscheint daher eher fraglich. Festzuhalten ist ferner, daß diese Schotter erst abgelagert werden konnten, als der Gletscher einen deutlich höheren Eisstand erreicht hatte. Bei dem Schottervorkommen N Weißlingen stellen wir außerdem ein tieferes Hinuntergreifen der Auflagerungsfläche auf der Molasse fest. Es hat darnach den Anschein, als wären diese Schotter nicht streng synchron mit denen in den andern zur Töß entwässernden Quertälern, sondern etwas jünger.

Dagegen sind die geschichteten Kiese von Bäretswil (cf. JuluUs Weber 1910) den Schottern der «hohen Terrasse»A. Webers altersgleich. Bei Neuegg werden sie von einem markanten Moränenwall gekrönt, den M. STEIN (1948) seinem «HofstetterSeeb» (= Schlieren)-Stadium zuweist. Da selbst die Wallmoränen des «Hohenstieglen» (= Wiedikon-Altstetten)-Stadiums noch den Schottern von Bäretswil aufsitzen, ist die Schotterflur der «hohen Terrasse» Armin Webers auch im Tal von BäretswilBauma nur als Vorstoßschotter eines etwa dem Zürich-Stadium konform verlaufenden frühwürmeiszeitlichen Eisstandes zu deuten. Dies wird durch analoge, verkittete und ebenfalls von Grundmoräne bedeckte Schotter auf dem Ricken bestätigt. A. Weber (1934: 37) stellt allerdings auch diese zur Hochterrasse und damit in die Riß-Eiszeit.

Etwas tiefer gelegene Schotter hat A. WEBER (1928) im oberen Tößtal der «mittleren Terrasse» zugewiesen und 1934 mit seiner Töß-Eiszeit in Beziehung gebracht ${ }^{2}$.

Während die Schotter der «hohen Terrasse» mit Ausnahme der beiden Vorkommen bei Weißlingen auf Grundmoräne ruhen, liegen die von A. WEBER im oberen Tößtal der «mittleren Terrasse»zugeordneten Schotter direkt auf Molasse. Im Bereich, wo die Schotterauflagerung nicht aufgeschlossen ist, die Niveaudifferenz zwischen der «hohen» und der «mittleren Terrasse» noch innerhalb der beiden Schotterkomplexe liegt, würde dieses tiefere Niveau ohne $Z$ weifel als «Erosionsterrasse» aufgefaßt. Dies ist jedoch nur bedingt richtig. Wohl erfolgte nach Ablagerung der «hohen Terrasse» eine ziemlich kräftige, bis auf die Molasse hinuntergreifende Eintiefung. WeBERs Schotter der «mittleren Terrasse» stellen aber, wie sich gerade im Tößtal klar feststellen läßt, eine neue Schüttung, also ein neues, auf eine Erosionsphase folgendes Akkumulationsniveau dar. Die Niveaudifferenz zwischen den beiden Akkumulationen beträgt bei Bauma rund $40 \mathrm{~m}$.

\section{DER GRENZBEREICH VON LINTH- UND RHEINSYSTEM}

\section{ZUR WÜRM-EISZEIT}

Es unterliegt keinem $Z$ weifel, daß die zur Töß entwässernden Quertäler bereits zur Riß-Eiszeit eingetieft worden waren; denn sonst hätten diese nicht von der anrückenden frühwürmeiszeitlichen Schotterflut erfüllt werden können. Daß es sich dabei kaum um Rückzugsschotter der Riß-Eiszeit handelt, hängt mit dem «Mechanismus» des Gletscherrückzuges zusammen. Dieser darf nicht einfach als das Rückwärtsablaufen eines Vorstoßes betrachtet werden. Durch die riß/würm-interglaziale Klimaverbesserung wurden nicht nur die Gletscherzungen, sondern der gesamte Eiskuchen betroffen. An den dünnsten Stellen, zwischen Glattal und Töß vor allem im Bereich der rißeiszeitlich flachgeschliffenen Wasserscheiden, schmolz das Eis durch, so daß zur ausgehenden Riß-Eiszeit die noch ins Tößtal hinüberreichenden Gletscherzungen als Toteis vom Stammgletscher abgetrennt wurden.

2 Das als Schotter der "mittleren Terrasse» ausgeschiedene Vorkommen von Niderau ESE Bauma gehört möglicherweise ins Niveau seiner "hohen Terrasse". 
Unmittelbar nach Ablagerung der frühwürmeiszeitlichen Schotterflur erfolgte im oberen Tößtal eine erste fluviatile Eintiefung durch die abfließenden Schmelzwässer, bot doch dieser Tallauf damals - zusammen mit einer alten, heute von Schotter erfüllten Rinne zwischen Kollbrunn und Seen (cf. J. Weber 1924 und A. Weber 1928) - den nordwestlichen Lappen des Linth/Rheingletschers und dem durch die Torsi von Flawil-Unterrindal, Littenheid-Anwil, Dußnang-Haslen und BichelseeTurbenthal entwässernden südlichen Bodensee-Rheingletschers, die einzige Abflußmöglichkeit in Richtung Hochrhein.

Während des ersten hochwürmeiszeitlichen Vorstoßes gelangte dann das etwas tiefere Schottersystem, dasjenige der «mittleren Terrasse» A. Webers zur Ablagerung. Diesem tieferen Schottersystem sind auch die beiden von A. WeBER ebenfalls der «hohen Terrasse» zugeordneten Schotterstränge im Tal von Gündisau und der Talung von Theilingen-Weißlingen zuzuzählen. Da ihr Ursprung auf der Glattalseite einen höheren Eisstand bedingt, konnten sie erst während des Vorrückens zum KillwangenStadium geschüttet werden. Dies konnte jedoch erst nach der ersten würmeiszeitlichen Erosionsphase geschehen - für das Tal von Gündisau, als der Gletscher über den Sattel $(718 \mathrm{~m})$ hinüberreichte - so ist es verständlich, daß die Auflagerungsfläche gegen die Töß hin tiefer auf die Molasse hinuntergreift. Die Schotter der «mittleren Terrasse» sind daher als Vorstoßschotter des in der Hochwürmeiszeit bis zum Killwangen-Stadium vorstoßenden Gletschers zu interpretieren. Dieses dürfte bei Weißlingen in den Stirnmoränen bei Widenwis vorliegen ${ }^{3}$.

Zufolge der vorgängigen Erosionsphase, die ohne Zweifel eine kleine frühwürmeiszeitliche Schwankung darstellt, liegen alle Schottervorkommen der «mittleren Terrasse» direkt der Molasse auf. Das Akkumulationsniveau liegt bei Bauma etwa $25 \mathrm{~m}$ über der Talsohle.

Nach Ablagerung der Schotter der «mittleren Terrasse» erfolgte zur Hochwürmeiszeit - bereits während des Killwangen-Stadiums und während des Rückzuges des Eises bis hinter die Wasserscheide von Gibswil - eine erneute Eintiefung. Diese reicht vom Akkumulationsniveau der «mittleren Terrasse» bis auf die Felssohle des Tößtales und dürfte bei Bauma rund $50 \mathrm{~m}$ betragen.

Die von A. Weber im oberen Tößtal als «Niederterrassenschotter und Schotter der Rückzugsstadien» ausgeschiedenen Talschotter stellen Schotter dar, die während des Zürich-Stadiums zur Ablagerung gelangten, als der Linth/Rheingletscher bei Gibswil noch einmal etwas ins Talsystem der Töß hinüber reichte. Da damals die Wasserscheide von Gibswil bereits flachgeschliffen war, kam es im Tößtal während des Spätund Postglazials nur zu einer geringen Eintiefung. Bei Steg beträgt diese, wie die ihres Fußes beraubten Schuttkegel dokumentieren, etwa 10-15 m. Je nach der Lage der Erosionsbasis wurde im Tößtal sogar akkumuliert. Dies ist darauf zurückzuführen, daß beim Abschmelzen des Gletschers bei Gibswil die Entwässerung ziemlich bald rückläufig erfolgen mußte. Dadurch wurde die bereits früher von rückläufigen Schmelzwässern angelegte Jonaschlucht zwischen Jonatal und Wald und zwischen Wald und Rüti - zufolge der während der Würmeiszeit erfolgten Tieferlegung der Erosionsbasis - noch weiter vertieft.

Die dargelegte Gliederung der Würm-Eiszeit, die sich sehr gut mit den von A. LeEmann (1958) im Rheintal zwischen Dießenhofen und Koblenz unterschiedenen Phasen deckt, äußert sich im Zürcher Oberland nicht nur in den Schotterterrassen, sondern auch in den Reliefformen: den weichen, glazial überschliffenen Formen des

${ }^{8}$ Die Karte (R. HantKe 1958, Taf. 2) ist zwischen Pfäftikersee und Töß dahin zu korrigieren, daß im Tößtal selbst die äußersten Würm-Endmoränen nicht bei Steg, wie damals auf Grund einer Bemerkung von J. FRÜH (1907) und J HUg \& A. BEILICK (1934) angenornmen wurde, sondern erst unterhalb Bauma liegen, was durch die Moränen von Hasenholz-Hörnen E Bauma und die Wälle N Nider Dürstelen und S Blitterswil dokumentiert wird. Bei Steg dürfte etwa das SchlierenStadium zu suchen sein. 
Glattales steht der fluviatil durchtalte Charakter des Tößtales und des Hörnligebietes scharf gegenüber. Als Grenze ergibt sich deutlich ein etwa dem Zürich-Stadium entsprechender Eisstand, bei dem der Linth/Rheingletscher bis Pfäffikon und Jona aufwärts bis Gibswil reichte.

Eine derartige morphologische Differenz ist, wie die Hohlformen der Alpenrandseen (cf. R. Hantke 1959b), kaum nur das Werk eines einmaligen Gletscherstandes - etwa dasjenige des hochwürmeiszeitlichen Zürich-Stadiums - sondern darin zeichnet sich das Erbe eines früheren, diesem konform verlaufenden Eisrandes ab.

\section{ECHTE HOCHTERRASSENSCHOTTER IM TÖSSTAL}

Es wäre nun aber verfehlt, sämtliche von A. WEBER seinerzeit als Schotter der «hohen Terrasse» ausgeschiedenen Vorkommen einfach als früh- bis hochwürmeiszeitlich zu betrachten.

Sicheren Hochterrassenschottern begegnen wir in seinem Untersuchungsgebiet vor allem außerhalb der äußersten Würm-Endmoränen, auf der rechten Seite des Rheines zwischen der Tößegg und Eglisau. Da die dort horizontal geschichteten Schottervorkommen stets ohne Grundmoräne der Molasse aufliegen und sie sich in ein bedeutend höheres Niveau einordnen lassen, ist eine Deutung als Vorstoßschotter der Riß-Eiszeit für diese Bildungen wahrscheinlich. Das Vorkommen von Nußbaumen NE Bülach ist ebenfalls diesem System zuzuordnen.

Fehlen nun im oberen Tößtal wirklich echte Hochterrassenschotter, also Vorstoßschotter der Riß-Eiszeit? Analog den Schottern im Bereich der Tößmündung - Auflagerungsfläche Murkatzelg (Niederterrasse) in $380 \mathrm{~m}$, Murkatfeld (Hochterrasse) in $440 \mathrm{~m}$ - müßte diese im oberen Tößtal mindestens ebenfalls $60 \mathrm{~m}$ höher liegen als die höchsten würmeiszeitlichen Schotter, als die wir bei Bauma WEBERs Schotter der «hohen Terrasse» mit einem Akkumulationsniveau von $710 \mathrm{~m}$ erkannt haben. Da gegen die Alpen überdies eine kräftigere Ausräumung der Täler erfolgte, ist dieser Wert sicher zu klein. Ein der Hochterrasse zuzuordnendes Schottervorkommen könnte allenfalls in der von A. WeBER auf der Nordseite des Stoffels festgestellten Schotterplatte vorliegen. Ihre Auflagerungsfläche auf der Molasse liegt knapp $100 \mathrm{~m}$ höher als diejenige der frühwürmeiszeitlichen Vorstoßschotters von Bliggenswil. A. WeBER betrachtet dieses Vorkommen - seiner Gliederung gemäß - zwar bereits als jüngeren Deckenschotter.

Nach unserem neuen Deutungsversuch würden naturgemäß auch die Gipfelkappe des Stoffels und die Vorkommen am Schloßkopf SW Steg und am Ghöch W Fischenthal ein «Stockwerk» tiefer rücken. Sie kämen damit ins System des jüngeren Deckenschotters zu liegen.

Durch diese neue Zuordnung ergäben sich für die beiden Schotterfluren, die frührißeiszeitliche Hochterrasse und die frühwürmeiszeitliche Niederterrasse, etwas steilere Gefällsverhältnisse, was anderseits auf eine etwas stärkere jungpleistozäne Hebung des Alpenkörpers schließen ließe.

\section{DIE DECKENSCHOTTER DER OSTSCHWEIZ}

Șehr wahrscheinlich dürften auch die beiden Deckenschottervorkommen am Tannenberg NW St. Gallen, Grimm und Etschberg, deren Geröliinhalt neulich von F. HormanN (1958) untersucht worden ist, nicht dem älteren (A. Gutzwiller 1900), sondern ebenfalls dem jüngeren Deckenschotter zuzuweisen sein, wie dies schon Roman FreI (1912a: 82/83) postuliert hat. Auch der Deckenschotter auf der Heid NE Wil (H. Wegelin und E. Gubler 1928) dürfte als jüngerer Dekkenschotter aufzufassen sein. Dagegen sind die beiden früher ebenfalls als Deckenschotter der Ostschweiz betrachteten Vorkommen des Bischofsberges und von Holenstein, S bzw. N von Bischofszell, sehr wahrscheinlich mit den Schottervorkommen im Haselwald S Roggwil und bei der Ruine Steinerberg und zusammen mit den Schieferkohle führenden Bildungen von Mörschwil, von Schönthal $N$ St. Gallen und von der Grüenegg WNW Bischofszell riß/würm-interglazial bis frühwürmeiszeitlich. 
All diese Schottervorkommen der Ostschweiz sollten jedoch unbedingt in größerem Zusammenhang geröllanalytisch und morphometrisch neu untersucht werden, umsomehr als im schwäbischbayrischen Alpenvorland auch noch glazifluviatile Schotter von prägünzeiszeitlichem Alter festgestellt wurden, von BARThEl EBERL (1930) die donaueiszeitlichen Hochschotter und von INGo SchÄFER (1953) ein noch älterer glazifluviatiler Komplex, der ihn zur Annahme einer ältesten Kaltzeit, der BiberEiszeit, geführt hat.

\section{DIE RISSEISZEITLICHEN BILDUNGEN IM GRENZBEREICH VON LINTH- UND RHEINSYSTEM}

Wenden wir uns nach diesem Abstecher wieder etwas sicherem Boden der Quartär-Chronologie zu und richten wir unser Augenmerk nochmals den rißeiszeitlichen Bildungen $\mathrm{zu}$.

Im Gebiet der Linthebene finden wir die höchsten Erratiker - zwei große und einige kleine Schrattenkalkblöcke - am Regelstein auf rund $1300 \mathrm{~m}$ Höhe (A. GutZwiler 1877: 126). Bei Möhlin im Aargau liegen die äußersten Moränenreste

der Riß-Eiszeit in rund $300 \mathrm{~m}$. Für die etwa $100 \mathrm{~km}$ messende Strecke RegelsteinMöhlin ergibt sich daraus ein durchschnittliches Gefälle der rißeiszeitlichen Gletscheroberfläche von rund $10 \%{ }^{4}$. Das Gebiet zwischen Pfäffikersee und oberem Tößtal lag somit damals offenbar tief unter dem Eis. Über dem vom Regelstein rund $20 \mathrm{~km}$ entfernten, $930 \mathrm{~m}$ hohen Stoffel dürfte damals die Eisdecke etwa bis $1100 \mathrm{~m}$ und über dem etwa $25 \mathrm{~km}$ entfernten, $820 \mathrm{~m}$ hohen Tämbrig bis $1050 \mathrm{~m}$ hinauf gereicht haben. Dagegen dürften der Gipfel des Hörnli und die Schnebelhorngruppe als Nunataker aus dem Eismeer des schweizerischen Mittellandes herausgeragt haben (cf. Roman FREI 1912b).

Am Stoffel begegnen wir den höchsten Moränenwällen in $840 \mathrm{~m}$, am Tämbrig in $799 \mathrm{~m}$ Höhe. Sie können somit auf keinen Fall dem Maximalstand der Riß-Eiszeit angehören. Hingegen dürfte dieses Niveau den höchsten Stand der Würm-Vereisung dokumentieren, ist doch ein Zusammenhang mit den Stirnmoränen in den zur Töß entwässernden Quertälern höchst wahrscheinlich, etwa zwischen der Moräne auf dem Isikerberg mit der Wallmoräne des Fischbacher Zungenendes.

Dagegen dürften die Wallmoränen von Chnüppis $(764 \mathrm{~m})$, Hintereggen $(750 \mathrm{~m})$ und Egg $(692 \mathrm{~m})$, alle im Raum Tambrig -Wildberg -Turbenthal, kaum mehr der Würm-Eiszeit angehören, sondern eine rißeiszeitliche Mittelmoräne darstellen.

\section{DIE UMGEBUNG VON WINTERTHUR ZUR WÜRM-EISZEIT}

Die Umdeutung der eiszeitlichen Bildungen im oberen Tößtal erfordern auch für die Umgebung von Winterthur eine Revision hinsichtlich ihrer quartärgeologischen Interpretation.

$\mathrm{N}$ Winterthur sind die Endmoränen von Andelfingen über die Wälle um die Nußbaumer Seen über den Seerücken ohne Schwierigkeit bis Stein am Rhein zu verfolgen, so daß der Gletscherstand von Andelfingen ohne $Z$ weifel dem Stadium von Stein am Rhein entspricht, das seinerseits stets mit demjenigen von Zürich parallelisiert wurde (cf. R. Hantke 1958). Anderseits ist der Zusammenhang der Endmoränen von Andelfingen über die Wälle um Dinhard über Bertschikon-Hagenbach mit den Moränen von Aadorf, Eschlikon und Gloten bei Wil gesichert. Die außerhalb südlich - dieses Stadiums gelegenen Moränen wurden nun aber von J. und A.Weber zwischen Aadorf und Wiesendangen bereits der Riß-Eiszeit zugeordnet, dagegen wird die ebenfalls außerhalb des Andelfinger Moränenkranzes gelegene Drumlinlandschaft N Winterthur der Würm-Eiszeit zugezählt. Auf der Übersichtskarte läßt A. WeBER

${ }^{4}$ Dieser Wert erscheint reichlich hoch. Wahrscheinlich ist ein Teil (vielleicht etwa $3 \%$ ) einer späteren, würmeiszeitlichen Hebung des alpennäheren Abschnittes zuzuschreiben. 
(1928, Taf. 1) den «Gletscherrand des Bülacher- und Rüdlinger-Stadiums» (= Killwangen) zuerst richtig längs dem Nordrand des Irchels folgen. Von Desibach an läßt dann WEBER die Eisrandlinie plötzlich ans Thurknie S Alten zurückspringen und gerät damit ins Schlieren-Stadium. Am E-Ende des Lindberges zeichnet er abermals eine merkwürdige Schlinge und gelangt bei Mörsberg ins System der Moränen des Zürich-Stadiums.

Zur Zeit des Würm-Maximums dürfte die Ebene von Winterthur noch bis über Pfungen hinaus vom Eis des Bodensee-Rheingletschers bedeckt gewesen sein. Dadurch wurde der durch das Kämttal abfließende Arm des Linth/Rheingletschers S Töß gestaut, so daß die Schmelzwässer gezwungen waren, durch den Torso Dättnau-Pfungen abzufließen. Da auch der alte Tößlauf von Kollbrunn nach Seen durch Rinnenschotter und Moränen des gegen S bis Iberg vorstoßenden Rheingletschers verstopft wurde, mußten sich die Schmelzwässer des Linth/Rheingletschers und des durch den Torso von Bichelsee ins Tößtal vorstoßenden Bodensee-Rheingletschers längs des südlichen Eisrandes des Rheingletschers einen neuen Abflußweg suchen. Die Anlage des Talstückes von Kollbrunn bis zur Einmündung der Kämt, sowie dessen nordwestliche Fortsetzung, der Torso Dättnau-Pfungen, dürften deshalb ins Hochwürm fallen.

\section{GLIEDERUNG DER RISS-EISZEIT BEI LAUFENBURG}

Wenn nun die «Töß», oder nach H. Suter (1939, 1944) die «Riß II-Schotter», früh- bis hochwürmeiszeitliche Vorstoßschotter darstellen, wie steht es dann mit der Gliederung der Riß-Eiszeit in «Riß I» und «Riß II»?

Um darüber etwas zu erfahren, werfen wir zunächst einen Blick auf ein von ED. BLösch (1911:6) veröffentlichtes Detailprofil von Schäffigen bei Laufenburg. Bei Fundierungen für den Kraftwerkbau konnte BlöscH in der tiefsten Baugrube über dem Gneis bis unter den Rheinspiegel hinunterreichende Schotter als Ausfüllung eines alten Tales feststellen. Der untere Komplex (1) ist im unteren Teil frisch, wird gegen oben schwach verwittert (2) ; dann folgt eine bis 1,4 m mächtige eigentliche Ver-

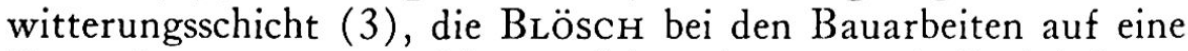
Erstreckung von etwa $4 \mathrm{~km}$ verfolgen konnte und die dabei nur auf kurze Strecken fehlte. Mit scharfer Grenze liegt darüber frische, schlecht geschichtete, kiesige Grundmoräne (4) mit zahlreichen großen Erratikern: Gneise und Ganggesteine der Umgebung, große geschliffene Blöcke von verkittetem Schotter, alpine und jurassische Blöcke sowie Tiefensteiner Granit und Porphyr.

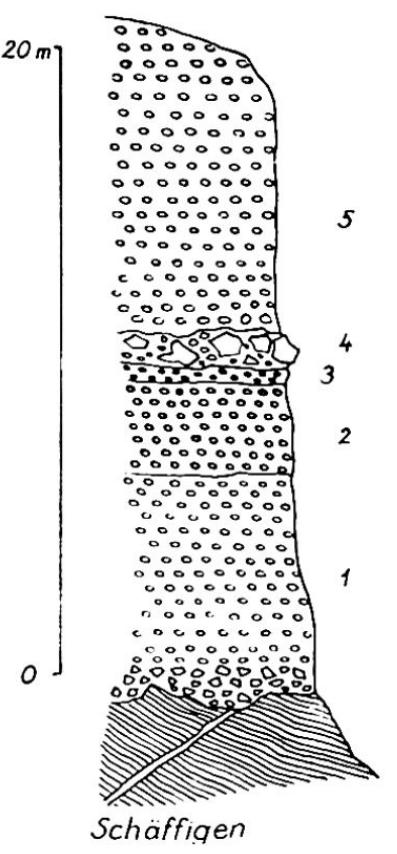
Diese Grundmoräne geht gegen oben ohne scharfe Grenze in einen höheren, jüngeren Schotterkomplex (5) über ${ }^{5}$.

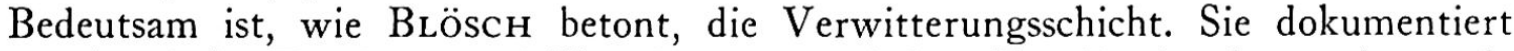
«eine subaerische Erosions- und Verwitterungsperiode». Da die darüber auftretende Grundmoräne nur von dem bis Möhlin vorgestossenen Riß-Gletscher abgelagert worden sein kann und der darunter liegende Schotter offensichtlich als rißeiszeitlicher Vorstoßschotter betrachtet werden muß, so bekundet die Verwitterungsschicht, wenn vielleicht nicht gerade wie $\mathrm{BLÖscH}$ meint, einen Beweis für ein Interglazial, so doch ein deutliches Interstadial.

Damit läßt sich die Riß-Eiszeit im Gebiet zwischen Laufenburg und Möhlin dank des vom Gletscher verschonten und in den Baugruben einst aufgeschlossenen Ver-

5 Die unteren Partien dürften wohl als hochrißeiszeitliche Rückzugsschotter zu deuten sein, während den höheren Partien spätrißeiszeitliches bis frühwürmeiszeitliches Alter zukommen wird. 
witterungshorizontes - in einen älteren Abschnitt mit Zungenende oberhalb Laufenburg (wie weit?), ein Frühriß, und einen Abschnitt mit bis Möhlin vorstoßendem Gletscherende, ein Hochriß, unterteilen.

Frühriß und Hochriß sind nun aber nicht genau identisch mit dem was in der schweizerischen Literatur bisher als «Riß I» und «Riß II» bezeichnet worden ist. Wie sich diese Gliederung weiter rheinaufwärts abzeichnet, bleibt noch zu untersuchen.

\section{RISSEISZEITLICHE RÜCKZUGSSTADIEN}

Da sich auch außerhalb der äußersten Würm-Endmoränen verschiedentlich - deutlich ausgebildete, meist aber ziemlich stark verwaschene Wallmoränen einstellen, ist es naheliegend diese als rißeiszeitliche Rückzugsstadien aufzufassen. Da sich derartige Wälle im Gebiet des rißeiszeitlichen Linth/Rheingletschers bei Siggenthal bereits in tiefen Lagen beobachten lassen, dürfte das Zungenende nicht mehr allzu entfernt gewesen sein.

Sehr wahrscheinlich ist damals auch der Reußgletscher und der durch dessen westliche Arme genährte Aare/Rhonegletscher ebenfalls nochmals bis gegen Lauffohr vorgestoßen. Ein seitlicher Schmelzwasserarm mag damals von Umikon über Rüfenach abgeflossen sein und die dortigen Hochterrassenschotter zerschnitten haben.

Auch im Wehntal stellen sich bei Niederwenigen Reste eines tiefliegenden Moränenwalles und damit Anzeichen eines synchronen Gletscherendes des Linth/Rheingletschers. Die jungpleistozänen Bildungen des Surbtales dürften daher etwas älter sein als dies E. BugmanN (1958) darlegt. Die Ablagerung des glazigenen Schotters von Chunte E Döttingen mit im Mittel 69\% gut gerundeten alpinen Geröllen dürfte in die Zeit dieses spätrißeiszeitlichen Gletscherstandes fallen; da von dem während des Würm-Maximums nur bis auf die Wasserscheide von Schöfflisdorf reichenden Gletscherlappen kaum ein derart hoher Anteil an Kristallingeröllen zu erwarten ist.

Unabhängig von diesen Untersuchungen konnte HERMANN EUgSTER bei seinen Kartierungsaufnahmen im östlichen Säntisgebirge und am Fänerenspitz zwischen den durch die höchsten Erratiker markierten Gletscherstand der Riß-Eiszeit und dem Maximalstand der Würm-Vereisung eine Häufung von Erratikern und verwaschene Wallreste beobachten und als zusammenhängendes Stadium verfolgen. Damit zeichnet sich auch in der Ostschweiz ein länger andauernder, spätrißeiszeitlicher Eisstand ab.

\section{LA SUBDIVISION DU PLEISTOCĖNE RÉCENT DANS LES RÉGIONS LIMITROPHES DU SYSTEME DE LA LINTH ET DU RHIN}

Après avoir reconnu dans la série des graviers de la terrasse moyenne (Mittelterrassenschotter) dans les vallées de la Glatt et du Lac de Zurich une succession de sédimentation complexe, commençant à la retraite de la glaciation rissienne et terminant avec l'avancée des glaciers wurmiens, ces dépôts ont été retrouvés récemment aussi dans la haute vallée de la Toess. ARMIN Weber (1928) les a mentionnés, à cause de leur position par rapport à la vallée, comme graviers de la haute terrasse et, par conséquent, il les a considérés d’âge rissien.

Ces cailloutis débutent dans les vallées transversales de la Toess du côté vers la Glatt à une altitude qui correspond à peu près à la surface du glacier de la Linth au stade de Zurich. Reposant sur une couche de moraine de fond assez épaisse, et étant couronnés par des vallums frontaux qui sont à attribuer au stade maximal de la glaciation wurmienne, cette série ne peut être que d'âge Wurm initial (Frühwürm).

Les cailloutis, considérés par WEBER comme graviers de la terrasse moyenne et attribués à sa glaciation aToess ( = Riss II d'après d'autres auteurs suisses) ont été reconnus comme dépôts du glacier wurmien qui avança après un petit recul interstadial, documenté par une période d'érosion, jusqu'au stade maximal.

Les cailloutis dans le fond de la vallée de la Toess, considérés par WEBER comme graviers de la basse terrasse et des stades de retraite, ont été déposé au moment ou le glacier de la Linth atteignit encore une fois le système de la Toess, c'est-à-dire pendant le stade de Zurich.

Dans les environs de Winterthour les bords du glacier du Rhin des trois stades wurmiens correspondants à ceux de Killwangen, de Schlieren et de Zurich ont été rectifiés dans leurs cours 
ce qui a permis de dater l'engorgement de l'ancien cours de la Toess entre Kollbrunn et Seen comme Wurm maximum. Le même âge résulte pour le parcours actuel entre Kollbrunn et l'embouchure de la Kaemt et pour le torso de Daettnau à Pfungen, car, à cette époque, c'était le seul thalweg qui a permis aux eaux des différents lambeaux du glacier de la Linth et du Rhin d'atteindre le Rhin wurmien à sa naissance, à la Toessegg.

Quant à la subdivision de la glaciation rissienne une coupe, dressée par BloEsCH (1911) pendant la construction du barrage de Laufenbourg, fournit des indications. BloEsCH a trouvé dans une série de graviers une couche extrêmement altérée qui était couverte de moraine de fond. Cette couche permet de distinguer un stade Riss initial (Frühriss) et un stade Riss maximum (Hochriss) pendant lequel le glacier avança jusqu’à Moehlin.

Les vallums morainiques de Siggenthal, situés très bas dans la vallée de la Limmat, font admettre que, à cette époque, le front du glacier de la Linth ne pouvait guère dépasser le verrou de Lauffohr. Ce même stade Riss tardif (Spätriss) parait se manifester au Wehntal dans un vallum près de Niederweningen.

Indépendamment H. Eugster a trouvé à l'Est des chaines du Saentis des moraines latérales intermédiaires entre le maximum de la glaciation Riss et $W$ urm qui ne peuvent guère documenter autre chose que ce stade Riss tardif.

\section{LITERATURVERZEICHNIS}

Baumberger, E. (1923): Die diluvialen Schieferkohlen in Dürnten (Kt. Zürich) - In: BaumBERGER, E. et alii: Die diluvialen Schieferkohlen der Schweiz - Beitr. Geologie Schweiz, Geotechn. Ser. 8. Bı.ösch, Ed (1911): Die Große Eiszeit - Beitr. geol. Karte Schweiz [NF] 3r/2. Bugmann, E. (1958): Eiszeitformen im nordöstlichen Aargau - Mitt. aarg. naturf. Ges. 25. Ebert, B. (1930): Die Eiszeitenfolge im nördlichen Alpenvorlande - Augsburg (Filser). FALKNer, C. (1910): Die südlichen Rheingletscherzungen von St. Gallen bis Aadorf - Jb. st. gall. naturw. Ges. (1908/09). FrEI, Romav (1912a): Monographie des schweizerischen Deckenschotters - Beitr. geolog. Karte Schweiz [NF] 37. - (1912b): Über die Ausbreitung der Diluvialgletscher in der Schweiz - Beitr. geol. Karte Schweiz [NF] ${ }_{4} r / 2$. FRÜH, J. (1907): Zur Bildung des Tößtales - Eclogae geol. Helv. o/3: 388. - (1919): Zur Morphologie des Zürcher Oberlandes - Vjschr. naturf. Ges. Zürich 6t: 16-34. Gutzwiller, A. (1877): Molasse und jüngere Ablagerungen, enthalten auf Blatt IX - Beitr. geol. Karte Schweiz [NF] $r_{4} / 1$. - (1900): Ältere diluviale Schotter in der Nähe von St. Gallen und von Bischofszell Eclogae geol. Helv. 6!4: 371-377. Hantke, R. (1958): Die Gletscherstände des Reuß- und Linthsystems zur ausgehenden Würmeiszeit - Eclogae geol. Helv. 5 r/1: 119-149. - (1959a): Zur Altersfrage der Mittelterrassenschotter. Die riß/würm-interglazialen Bildungen im Linth/Rheinsystem und ihre Äquivalente im Aare/Rhone-System - Vjschr. naturf. Ges. Zürich Iof/1: 1-47. - (1959b): Zur Phasenfolge der Hochwürmeiszeit des Linth- und des Reuß-Systems, verglichen mit derjenigen des Inn- und Salzach-Systems sowie mit der nordeuropäischen Vereisung - Vjschr. naturf. Ges. Zürich 104: 390-402. Heer. O. (1858): Die Schieferkohlen von Uznach und Dürnten - Zürich (Orell-Füssli). - (1865): Die Urwelt der Schweiz - Zürich (Schulthess), 2. Aufl. 1879 (ersch. 1883). Hess, E. (1946a): Exkursion Nr. 16/2: Winterthur-Eschenberg-Kyburg-First-Kempttal - In: Geologische Exkursionen in der Umgebung von Zürich - Geol. Ges. Zürich, Zürich (Leemann). (1946b): Exkursion Nr. 17: Elgg-Aadorf-Wil-Heid - Ibid. Hofmann, F. (1951): Zur Stratigraphie und Tektonik des st. gallisch-thurgauischen Miozäns (Obere Süßwassermolasse) und zur Bodenseegeologie - Jb. st. gall. naturw. Ges. 74: 3-87. - (1958): Pliozäne Schotter und Sande auf dem Tannenberg NW St. Gallen - Eclogae geol. Helv. 5o/2 (1957): 477-482. Huber, R. (1956): Ablagerungen aus der Würmeiszeit im Rheintal zwischen Bodensee und Aare - Vjschr. naturf. Ges. Zürich ror/1: 1-92. Hug, J. (1907): Geologie der nördlichen Teile des Kantons Zürich und der angrenzenden Landschaften - Beitr. geol. Karte Schweiz NF. 15. - (1917): Die letzte Eiszeit der Umgebung von Zürich - Vjschr. naturf. Ges. Zürich 62: 125-142. - (1932): Zur Gliederung der Hochterrasse im Limmat- und Glattal - Eclogae geol. Helv. 25/2: 264-265. Hug, J. \& Berl.iCK, A. (1934): Die Grundwasserverhältnisse des Kantons Zürich - Beitr. Geologie Schweiz, Geotechn. Ser., Hydrol. I. Knauer, J. (1938): Über das Alter der Moränen der Zürich-Phase im LinthgletscherGebiet - Abh. geol. Landesuntersuch. Bayer. Oberbergamt 33, München. Leemans, A. (1958): Revision der Würmterrassen im Rheintal zwischen Diessenhofen und Koblenz - Geogr. Helv. 13/2: 89-173. LÖDI, W. (1953): Die Pflanzenwelt des Eiszeitalters im nördlichen Vorland der Schweizer Alpen - Veröff. Geobot. Inst. Rübel 27. Ludwig, A. (1920): Ältere Schotter in der Umgebung von St. Gallen - Jb. st. gall. naturw. Ges. (1919): 38-39. Mühlberg, F. (1896): Der Boden von Aarau - Festschr. Einweihung Kantonsschulgebäude Aarau, Aarau (Sauerländer). Penck, A. und Brückner, E. (1909): Die Alpen im Eiszeitalter, 2 - Leipzig (Tauchnitz). SCHäFer, I. (1953): Sur la division du Quaternaire dans l'avant-pays des Alpes en Allemagne - Actes INQwA IV, Rom $I$. Staub, R. (1939): Prinzipielles zur Entstehung der alpinen Kandseen - Eclogae geol. Helv. 31/2: 239-258. Stein, M. (1948): Morphologie des Glattales - Diss. Univ. Zürich, Uster. Suter, H. (1939): Geologie von Zürich - Zürich (Leemann). - (1944): Glazialgeologische Studien im Gebiet zwischen Limmat, Glatt und Rhein - Eclogae geol. Helv. 37/1: 83-97. Weber, A. (1928): Die Glazialgeologie des Tößtales und ihre Beziehungen zur Diluvialgeschichte der Nordostschweiz - Mitt. naturw. 
Ges. Winterthur ${ }_{77} /{ }_{1} 8$ (1927-1930), als Diss. 1928. - (1934): Zur Glazialgeologie des Glattales Eclogae geol. Helv. 27i1: 33-43. - (1946): Exkursion Nr. 13: Uster-Wetzikon-Dürnten-BäretswilStoffel-Hittnau-Pfäffikon - In: Geologische Exkursionen in der Umgebung von Zürich - Geol. Ges. Zürich, Zürich (Leemann). Weber, J. (1901): Beiträge zur Geologie der Umgebung des Pfäffikersees - Mitt. naturw. Ges. Winterthur 3. - (1924): Erläuterungen zur geologischen Karte der Umgebung von Winterthur 1:25000 - Geol. Karte Schweiz 2I u. Mitt. naturw. Ges. Winterthur 15. Wegelin, H. und Gubler, E. (1928): Deckenschotter auf der Heid - Mitt. thurg. naturf. Ges. 27. Wetrstein, A. (1885): Geologie von Zürich und Umgebung, mit geol. Karte 1:40000 Diss. Univ. Zürich, Frauenfeld (Huber). Woldstedr, P. (1958): Das Eiszeitalter. Grundlinien einer Geologie des Quartärs, 2 - Stuttgart (Enke).

\section{LOCALITÉS SUISSES TIRANT LEUR ORIGINE DE DOMAINES GALLO-ROMAINS Maurice-Ed. Perret}

Jules César, dans ses Commentaires de la guerre des Gaules, raconte la tentative d'émigration des Helvètes. Ceux-ci, battus par les Romains, durent rentrer dans leur pays et rebâtir les douze villes et les quatre cents villages qu'ils avaient incendiés avant de partir. Nulle part ne figure la liste des douze villes et l'on est réduit à des hypothèses sur leur identification; quant aux villages, on n'en sait presque rien. L'on n'a pas de détails sur la manière dont les Romains occupèrent le pays. Dans toute la Gaule, ils créèrent des domaines agricoles, au centre de chacun desquels s'élevait un groupe de bâtiments, la villa. Les villas primitives se sont généralement agrandies et sont devenues des villages. Lorsque le pays a été christianisé, c'est auprès de la villa que l'on a construit une chapelle et ainsi nombre de villas sont devenues des communes et des paroisses.

Le domaine gallo-romain, en latin fundus, était désigné par un adjectif dérivé du nom de famille romain (gentilice) ou d'un nom d'individu romain ou gaulois. Ainsi le domaine d'une famille Colonius se nommait fundus coloniacus, simplifié en Coloniacum, aujourd'hui Cologny. Par suite de l'évolution de la langue, la terminaison -acum a suivi un sort différent selon les régions de la Gaule. En Suisse romande, après avoir pris au moyen âge la forme -ie, elle est devenue soit $-y$ (Montagny de Montanius), -ex (Saconnex de Saconnus), -ez (Chevenez de Cavinus), -er (Auvernier de Avernius), plus rarement -ay (Chavornay de Cavorinus), -ey (Chabrey de Cabrus); dans les régions alémaniques -acum est devenu -ach (Alpnach de Alpinus).

Le fait que le nom d'une localité se termine par $-y,-e x,-e z,-e r,-a y,-e y$ ou $-a c h$, n'est pas suffisant pour être une preuve certaine d'origine gallo-romaine. Beaucoup de noms terminés ainsi sont moins anciens; par exemple $A c h$ en allemand ancien désignait un cours d'eau et la plupart des noms où l'on retrouve cette terminaison n'ont pas de relation avec les Gallo-Romains. On peut cependant trouver d'autres preuves. Tout d'abord, des fouilles archéologiques ont pu mettre à jour des vestiges de villa galloromaine ou simplement des objets d'époque romaine; ainsi les restes d'une importante villa à Alpnach. Dans certains cas, des documents du moyen âge indiquent des formes anciennes de localités dans lesquelles on reconnaît sans hésitation un nom romain et la terminaison -acum. Dans certains noms modernes, on peut facilement déceler un nom de famille ou d'individu, romain ou gaulois qui se retrouve dans d'autres noms de lieux; certains gentilices étaient très répandus, ainsi Florius est reconnaissable dans les noms de plus de cinquante localités de l'ancienne Gaule, c'est de lui que vient chez nous Fleurier. Enfin, la situation géographique permet l'identification de nombreux domaines gallo-romains: en effet, les villas romaines n'ont pas été placées au hasard; les villages actuels qui leur ont succédé sont habituellement dans de belles situations, bien exposés au soleil, entourés de champs fertiles; on ne les trouve pas dans les bas-fonds sujets aux brouillards, aux inversions de température, aux gelées d'hiver, ni près des marais, ni au bord des lacs ou des rivières qui peuvent déborder, ni sur les crêtes balayées par les vents, ni sur des pentes escarpées, mais, partout où cela est possible, sur un replat, sur une petite éminence au-dessus de la plaine, du lac ou du fond de la vallée; enfin, elles sont situées en général dans le voisinage des villes gallo-romaines ou à proximité de routes romaines. 